Background Escalation planning including decisions regarding CPR and higher levels of care should be made in advance wherever possible. A Treatment Options Form was devised in Sheffield to promote and aid the documentation of escalation planning for patients with life limiting conditions. We sought to increase the use of this form within a tertiary oncology centre, with the hope of improving the number of timely advance care decisions.

Methods We included 135 patients from August 2016 to May 2017. We completed data collection at baseline and then a further four data cycles after each of the following interventions:

- introduction of form into clerking pack for new patients

- training to new junior doctors at induction

- presentation to consultants and questionnaire to glean barriers to usage of form

- ensured all patient notes contained a treatment option form.

We looked at all discharges within the pre-defined data collection window (1 week per data cycle), including only those who had originally been admitted as an emergency. The notes were analysed retrospectively and data analysed using excel.

Results Documentation of escalation plans increased from 13\% - 52\%; of these, discussions with relatives increased from $50 \%-83 \%$. Setting of these plans within 24 hours of admission increased from $7 \%-13 \%$ and use of the Treatment Options Form increased from $7 \%$ - 48\%. Feedback from questionnaires suggested that barriers to implementing advance planning included time pressures and reluctance of acute teams to make decisions for patients not under their outpatient follow up.

Conclusions Implementing a Treatment Options Form has improved the numbers of advance care decisions for patients in the oncology setting. We have identified some of the barriers to these decisions being made. Further work to elucidate how best to overcome these is required.

\section{A NEW START: REDESIGNING A NOVEL SUPPORTIVE AND PALLIATIVE CARE SERVICE IN AN ACUTE HOSPITAL TRUST}

Jayne Winter, Farida Malik, David Barclay. East Sussex Healthcare NHS Trust

\subsection{6/bmispcare-2018-ASPabstracts. 165}

East Sussex Healthcare Trust (ESHT) is a combined community/ acute hospital trust, with two acute hospitals 20 miles apart and 100000 admissions a year. In light of workforce changes, the need for joint working across hospital teams and CQC inspection results, a new Supportive and Palliative Care service (SPC) was developed to improve care delivered.

Methods An away day with key stakeholders was organised Dec 2016 to highlight needs of future service and standardise working practices between members of palliative care and EOLC team's cross-site. Both teams amalgamated to become Supportive and Palliative Care Team (CNSs, PDNs, consultants and a specialty doctor). A new nurse team leader role was created and medical clinical lead appointed. Clear unified SPC referral guidelines were developed with one referral point of contact mobile number on each site being used. A standardised cross-site operating procedure was developed. Video conferenced weekly MDT meetings are held cross-site.

An awareness campaign, including newsletter and team information sheet has been developed. The team are involved in multidisciplinary teaching delivery and regular team and governance meetings. Team KPI's are presented to the Trust EOLC steering group. An online staff feedback survey has been developed and will go out to all staff to influence team development (results to follow).

Results From January - September 2017 there's been a 63\% increase in referrals to one site, with a $30 \%$ increase crosssite. $90 \%$ of patients are seen within 24-hours of referral with $60 \%$ reviewed same day. $30 \%$ of the caseload is non-malignant, a $10 \%$ increase. The team supports all patients identified as being in their last days of life on an individualised care plan (fully embedded in practice).

Conclusion There has been an improvement in cross-site hospital team working. Staff feedback on the team will further influence team development.

\section{9 'PREFERRED PLACE OF DEATH' IN PALLIATIVE CARE PATIENTS -'RE-AUDIT' USING DATA COLLECTED AT THE ROYAL SURREY COUNTY HOSPITAL MAY - JUNE 2017}

Liam Gabb, Sophie Norman. Royal Surrey County Hospital

\subsection{6/bmjspcare-2018-ASPabstracts. 166}

Choice surrounding place of death (PPD) is a fundamental component of a good death, with NICE quality standards for end of life care stating that all patients should have their needs and preferences regarding this assessed. In an initial audit to determine whether RSCH palliative care inpatients had had an opportunity to discuss preference for place of death, results showed we did not meet our standard (set at $100 \%$ of patients having had this opportunity, or a valid reason documented as to why not). Less than $50 \%$ had a documented PPD, and less than 75\% had a valid reason why not. To complete the audit cycle, this re-audit looked at outcomes following changes made to assessment documentation and team education, and compared to the initial results.

Data collected from palliative care inpatients May 2017 June 2017 (N=226) regarding PPD documentation, and compared to initial data October 2016 - January 2017 ( $N=274)$.

The target of recording a PPD or a valid reason for its omission was achieved in 168 (88.4\%) patients, a slight increase compared to initial audit (84.5\%). For patients who died, death at their PPD was achieved in $76.0 \%$ - an increase from $67.6 \%$.

Though not meeting it, outcomes in the second cohort of patients were closer to the set standard than in our initial data, suggesting successful intervention. Mandatory documentation and discussion of PPD earlier in the healthcare journey are further suggestions to improve this. Despite commenting on numbers of patients meeting their PPD, we did not develop or analyse this information further and feel this could be a point for further audit or QIP. 